\title{
Dank FMH Services zu günstigeren Konditionen einkaufen!
}

Wir freuen uns, Ihnen dieTeva Pharma AG als unseren neuen Zusammenarbeitspartner im Zentralen Einkauf für Ärztinnen und Ärzte vorstellen zu dürfen.

Teva steht für Generika. Und für noch viel mehr. Denn mit ihrer Philosophie, stets mehr zu leisten, sind sie zu einem international aktiven Pharmaunternehmen mit mehreren Standbeinen herangewachsen. In ihrer eigenen Forschungs- und Entwicklungsabteilung arbeiten sie erfolgreich an wegweisenden Produkten und innovativen Wirkstoffen für die Gesundheit der Zukunft. Und weil sie Gesundheit nicht nur erhalten, sondern auch erschwinglich machen wollen, stehen sie mittlerweile an der Spitze der weltweit führenden Generika-Hersteller. Mit diesen Kompetenzen steht Ihnen die Teva als verlässlicher Partner auf dem Schweizer Pharmamarkt zur Seite. Mehr überTeva erfahren Sie unter www.tevapharma.ch.

Sie haben als Genossenschafter/in der FMH Services die Möglichkeit, Ihre zukünftigen Bestellungen bei derTeva Pharma AG direkt über ein Sammelrechnungs-System abrechnen zu lassen. Nebst der Ersparnis von Spesen und administrativem Aufwand profitieren Sie von Sonder- und Exklusivangeboten in Rabattform.

\section{Ihre Vorteile}

- 7\% Rabatt auf allen Einkäufen bei derTeva Pharma AG für Neukunden. Wenn Sie Ihre Einkäufe zum ersten Mal über die FMH Consulting Services abrechnen lassen, profitieren Sie im ersten Jahr von 7\% Rabatt. Ab dem 2. Jahr sind es $5 \%$.

- einmalige, monatliche Rechnung für alle Bezüge bei Vertragslieferanten während der letzten 4 bis 6 Wochen;

- grösseres Zahlungsziel, geringerer administrativer Aufwand und weniger Bankspesen.

Haben wir Ihre Neugierde geweckt? Wenn Sie sich an unser Sammelrechnungs-System anschliessen möchten, bitten wir Sie, uns den unten angefügten Antworttalon zu retournieren. Sie erhalten dann das Bestellformular in elektronischer Form oder per Fax und können direkt beiTeva bestellen. Die monatliche Rechnung wird durch FMH Consulting Services erstellt.

Wir freuen uns, in Teva Pharma AG einen kompetenten Einkaufspartner gefunden zu haben.

\section{Sammelrechnung}

\section{Fax 0419210586 oder E-Mail: mail@fmhservices.ch}

Im Sinne einer optimalen Ausnutzung dieses speziellen Angebotes der FMH Consulting Services möchte ich meine zukünftigen Einkäufe bei derTEVA Pharma AG über das Sammelrechnungs-System abrechnen lassen.

Ich nehme davon Kenntnis, dass die Dienstleistung Sammelrechnung über die FMH Consulting Services für mich kostenlos ist.

Name:

Strasse:

Tel. privat:

ZSR-Nummer:

(ehem. Konkordats-Nr.)
Vorname:

PLZ/Ort:

Tel. Geschäft:

E-Mail: 